\title{
Rhinosinusitis: Bioflavonoide hemmen die Entzündung und steigern die Sekretolyse
}

— Die Pflanzenkombination Sinupret ${ }^{\circledR}$ ist ein häufig zur Behandlung der akuten und chronischen Sinusitis eingesetztes Präparat - zu Recht, wie aktuelle Studien belegen. Das Phytopharmakon hemmt nämlich verschiedene Entzündungsmediatoren und steigert die Sekretolyse. Ermöglicht wird dies durch die zahlreich enthaltenen Bioflavonoide.

Die sekretolytische Wirkweise beruht auf der Erhöhung der Permeabilitiät des CTFRKanals [Virgin F, Zhang S, Schuster D, Azbell C, Fortenberry J, Sorscher E J, Woodworth B
A. The bioflavonoid compound, sinupret, stimulates transepithelial chlorid transport in vitro and in vivo. The Laryngoscope 2010; 120: 1051-6].

Ein weiteres wichtiges Therapieziel neben der Sekretolyse und der Wiederherstellung der mukozilliären Clearance ist die Eindämmung der überschießenden Entzündungsreaktion des Immunsystems. Sinupret ${ }^{\circledR}$ besitzt durch die Inhibition verschiedener Entzündungskaskaden und Entzündungsmediatoren und die Fähigkeit, die Konzentration proinflammatorischer Zytokine zu reduzieren, eine breit angelegte antiinflammatorische Wirkung. Die pflanzliche Wirkstoffkombination unterbindet die wichtige Immunreaktion des Körpers aber nicht vollständig, sondern dämmt lediglich die überschießende Entzündungsreaktion ein.

Die antiinflammatorische Wirkweise ergänzt die bekannte sekretolytische Wirkung von Sinupret ${ }^{\circledast}$ durch die sich der festsitzende Schleim löst und die Nase öffnet, weil die Schwellung der Nasenschleimhat zurückgeht. Ausgeprägte antivirale bzw. antibakterielle Eigenschaften des Phytotherapeutikums runden das pharmakologische Wirkspektrum ab.

$g z$

Nach Informationen von Bionorica SE

\section{Neuer Patientenflyer „Halsbeschwerden"}

- Halskratzen und Heiserkeit sind häufige Gründe für einen Besuch beim Arzt. Oft schuld daran: Trockene Raumluft, stundenlanges Sprechen oder ein Atemwegsinfekt lassen den feinen Sekretfilm auf der Rachenschleimhaut versiegen, der die empfindliche Schleimhaut vor Krankheitserregern und Schadstoffen schützt.

Die Frage "Was hilft bei Halsbeschwerden" beantwortet der neue Patientenflyer von Pohl-Boskamp. Neben einer Redepause, dem Verzicht auf Alkohol und Zigaretten bieten GeloRevoice ${ }^{\circledast}$ Halstabletten mit Hyaluronsäure zuverlässige Hilfe. Beim Lutschen setzen sie ein hochwertiges Hydrogel auf der Schleimhaut frei, das Mund- und Rachen nachhaltig befeuchtet. Schon nach wenigen Tagen Behandlung klingen Halsbeschwerden um rund $60 \%$ ab, zeigte jetzt eine Beobachtungsstudie. Mehr als $80 \%$ der Ärzte benoteten die Wirksamkeit der Therapie mit "sehr gut" oder "gut".

Zu bestellen ist der Patientenflyer bei:

G. Pohl-Boskamp GmbH \& Co. KG

Unternehmenskommunikation

Kieler Straße 11

25551 Hohenlockstedt

Nach Informationen von Pohl-Boskamp

\section{Günstigere Therapie mit mometasonhaltigen Nasensprays}

— Zur Behandlung der allergischen Rhinitis werden häufig kortikoidhaltige Nasensprays eingesetzt. Meist werden wirkstoffhaltige Nasensprays mit Mometason (MHN) oder Budesonid (BHN) verordnet, die in den entsprechenden Formulierungen zum Teil unterschiedliche Dosierungsempfehlungen haben. Um den Verbrauch und damit die in Folge auftretenden Kosten von unterschiedlichen Medikamenten zu bestimmen, wurden basierend auf der IMS ${ }^{\circledR}$ Disease Analyzer Datenbank für Deutschland Erstverordnungen aus dem Zeitraum August 2006 bis Juli 2010 ausgewertet, für die mindestens eine Folgekonsultation dokumentiert war. Auf der Basis der seit Juli 2011 gültigen Apothekenpreise wurden daraus die entsprechenden Jahrestherapiekosten berechnet.

Zur Auswertung wurden die Daten von Patienten aus Allgemeinarzt-Praxen (15.907 mit MHN und 4.006 mit BHN behandelte Patienten) und HNO-Praxen (10.660 MHN und 2.335 $\mathrm{BHN}$ ) herangezogen: Die Verordnungsmengen zeigten deutliche Unterschiede sowohl in den Allgemeinarzt-Praxen als auch in den HNO-Praxen: So verordneten HNO-Ärzte zu Beginn der Therapie bei MHN 105 Hübe pro Patient, bei BHN 242,3 Hübe. Nach zwei Jahren stieg die Differenz weiter an (305,1 Hübe gegen 793,4 Hübe $p<0,0001)$. Bei den Allgemeinärzten zeigte sich ein ähnliches Bild.

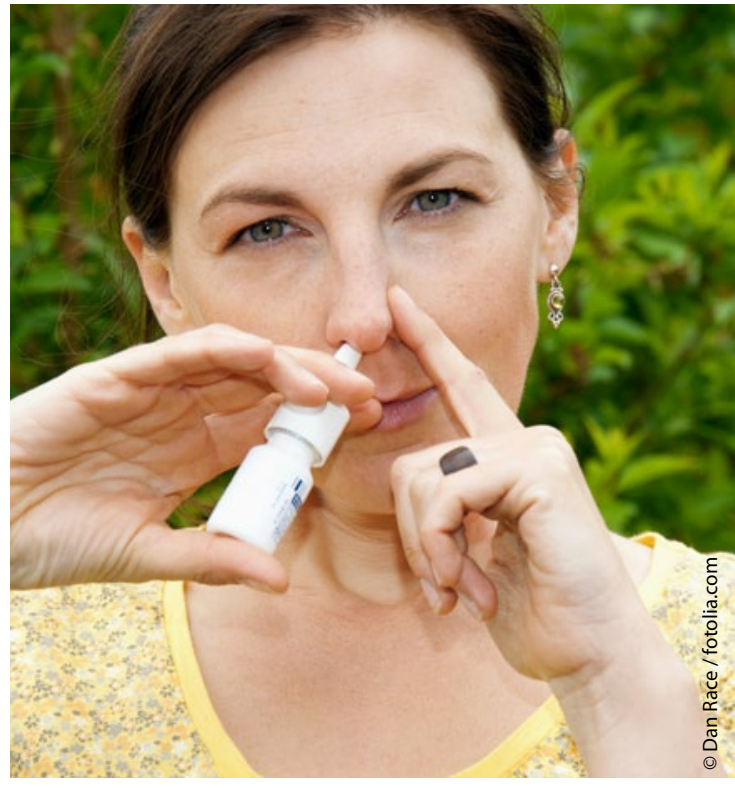

Nach Verknüpfung der unterschiedlichen Verordnungsmengen mit den seit Juli 2011 aufgrund der Festbetragsanpassung gesenkten Apothekenverkaufspreisen wurden für HNO-Praxen als mittlere Medikamentenkosten pro Jahr und Patient 22 Euro für MHN (Nasonex ${ }^{\oplus}$ ) und 29,70 Euro für BHN errechnet.

$g z$

Nach Information von MSD 